\title{
Identification of potential drug targets based on a computational biology algorithm for venous thromboembolism
}

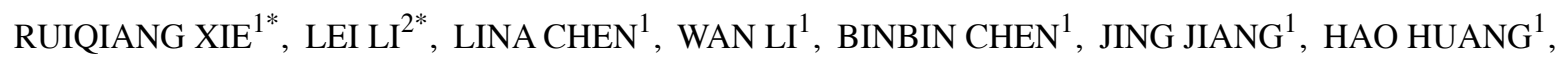 \\ YIRAN LI ${ }^{1}$, YUEHAN HE ${ }^{1}$, JUNJIE $\mathrm{LV}^{1}$ and WEIMING HE ${ }^{3}$ \\ ${ }^{1}$ College of Bioinformatics Science and Technology, Harbin Medical University; \\ ${ }^{2}$ The Second Affiliated Hospital of Harbin Medical University, Harbin, Heilongiiang 150081; \\ ${ }^{3}$ Institute of Opto-electronics, Harbin Institute of Technology, Harbin, Heilongjiang 150086, P.R. China
}

Received January 25, 2016; Accepted December 9, 2016

DOI: $10.3892 /$ ijmm.2016.2829

\begin{abstract}
Venous thromboembolism (VTE) is a common, fatal and frequently recurrent disease. Changes in the activity of different coagulation factors serve as a pathophysiological basis for the recurrent risk of VTE. Systems biology approaches provide a better understanding of the pathological mechanisms responsible for recurrent VTE. In this study, a novel computational method was presented to identify the recurrent risk modules (RRMs) based on the integration of expression profiles and human signaling network, which hold promise for achieving new and deeper insights into the mechanisms responsible for VTE. The results revealed that the RRMs had good classification performance to discriminate patients with recurrent VTE. The functional annotation analysis demonstrated that the RRMs played a crucial role in the pathogenesis of VTE. Furthermore, a variety of approved drug targets in the RRM M5 were related to VTE. Thus, the M5 may be applied to select potential drug targets for combination therapy and the extended treatment of VTE.
\end{abstract}

\section{Introduction}

Venous thromboembolism (VTE) is a common and chronic disease with a considerable risk of recurrence and can be fatal $(1,2)$. The crucial steps to preventing further complications and recurrence are an accurate diagnosis and early treatment

Correspondence to: Professor Lina Chen, College of Bioinformatics Science and Technology, Harbin Medical University, 157 Baojian Road, Harbin, Heilongjiang 150081, P.R. China

E-mail: chenlina@ems.hrbmu.edu.cn

Professor Weiming He, Institute of Opto-electronics, Harbin Institute of Technology, 92 Xidazhi Street, Harbin, Heilongjiang 150086, P.R. China

E-mail: hewm@hit.edu.cn

*Contributed equally

Key words: human signaling network, venous thromboembolism, recurrent, drug targets with appropriate drugs (3). Biomarkers have been specifically investigated for their capacity to predict VTE during the course of disease (4). The analysis of VTE-related molecular expression may identify potential disease specific biomarkers associated with disease recurrence and as potential drug targets (5). The expression of the potent pro-coagulant protein tissue factor can trigger thrombosis (6). Although a number of putative mechanisms have been proposed, the mechanisms that initiate VTE have not been completely elucidated, and treatment options for individuals with recurrent disease in particular remain limited (7). The understanding of the mechanisms of VTE may lead to the development of effective treatments for patients. Accurate diagnosis, early prevention and the treatment of VTE with appropriate drugs are crucial to prevent further complications and recurrence (3). Warfarin and low molecular weight heparin (LMWH) are the conventional drugs used for the treatment of recurrent VTE. Kearon suggested that warfarin was effective in the treatment of recurrent VTE (8). However, the use of warfarin for anti-coagulation in patients with vein thrombosis does not minimize the risk of recurrence (9). Thus, more systematic research and drug combination strategies have been recommended (10).

Network-based methods have been implemented to predict drug side-effects, drug targets and new therapeutic indications (11). The biological functional network modules can reveal disease mechanisms and identify drug targets (12). The dynamic changes in gene expression would improve the understanding of the pathological processing of complex diseases. The analysis of gene expression using the network may be more effective in predicting drug targets (13). According to the different factors and differential gene expression in easily recurrent thrombosis, a systemic method for screening drugs and research on recurrent VTE may prove to be very good strategy (14). The integrated analysis of the network and high-throughput data is benefiical to human disease research. In this study, we developed a novel module screening method to identify the dynamic module changes by combining human signaling networks and gene expression. We aimed to elucidate the molecular mechanisms responsible for recurrent VTE, and identify drug response pathways and potential drug targets with which to improve the efficacy of VTE therapy and reduce the recurrence of VTE. 


\section{Materials and methods}

Data source. The human signaling network data were obtained from previous studies $(15,16)$. This included 6,287 genes and 62,239 signaling relations. The human signaling network was integrated into the human subcellular signaling networks (HSSNs) from the Gene Ontology (GO) database (17) and Universal Protein Resource (UniProt) (version UniProt release 2014_03) (18).

We obtained the whole blood gene expression profile dataset GSE19151 from the Gene Expression Omnibus database. The GSE19151 expression profile dataset contains 3 sets of samples, including 70 adults with one or more prior VTE treated with warfarin (32 single and 38 recurrent) and 63 controls. The samples were collected and screened by the Duke Anticoagulation Satisfaction Scale (DASS) (19). In the study, patients over 18 years had at least one prior venous thromboembolic event. All patients administered warfarin were had suffered from their most recent thromboembolic event $\geq 4$ weeks earlier. Patients who were just administered an anticoagulant (warfarin) and patients with cancer or antiphospholipid syndrome were excluded. The investigators who enrolled all VTE events reviewed and confirmed the number of events, thrombus location, thrombus type, and other clinical data. This study included patients who suffered from spontaneous or provoked VTE for exploratory research from a previous study (20). Blood was collected in PAXgene tubes followed by RNA extraction and gene expression profiling by Affymetrix arrays. The independent VTE expression profile datasets GSE17078 from GPL96 platform contained 3 samples of patients with VTE (VT sample) and 27 normal samples.

Recurrent risk modules (RRMs). Considering the different conditions of recurrent VTE under the treatment of warfarin, we developed a novel algorithm for identifying RRMs. RRMs were selected by two steps: first, we determined three different stages (control/single, control/recurrent and single/ recurrent) modules, respectively. Network modules were screened through an efficient Markov clustering algorithm of the GraphWeb (http://biit.cs.ut.ee/graphweb/) tool in each HSSN (21). The default value of 1.8 for the Markov clustering parameter was considered. Modules with less than four genes were excluded. The genes in each module were both highly correlated and topologically close. The differential stage modules were then screened by evaluating the expression correlation differential score, which represented the significantl changes in expression in different sets of samples at different stages. Supposing a module M contains $\mathrm{m}$ edges E1...Em from the HSSNs, thus the differential score $\mathrm{S}$ was expressed as follows:

$$
\begin{gathered}
S(M)=\sum_{k=1}^{m}\left|E_{k}-E_{k}{ }^{\prime}\right| \\
E_{k}=\operatorname{pearson}(X, Y)=\frac{\sum(X-\bar{X})(Y-\bar{Y})}{\sqrt{\sum_{i=1}^{n 1}\left(X_{i}-\bar{X}\right)^{2}} \sqrt{\sum_{i=1}^{n 1}\left(Y_{i}-\bar{Y}\right)^{2}}} \\
E_{k}{ }^{\prime}=\operatorname{pearson}\left(X^{\prime}, Y^{\prime}\right)=\frac{\sum\left(X^{\prime}-\bar{X}^{\prime}\right)\left(Y^{\prime}-\bar{Y}^{\prime}\right)}{\sqrt{\sum_{i=1}^{n 2}\left(X_{i}^{\prime}-\bar{X}^{\prime}\right)^{2}} \sqrt{\sum_{i=1}^{n 2}\left(Y_{i}^{\prime}-\bar{Y}^{\prime}\right)^{2}}}
\end{gathered}
$$

wherein, $\mathrm{X}, \mathrm{Y}$ and $\mathrm{X}, \mathrm{Y}^{\prime}$ denote the gene expression data of two distinct stages, respectively. $\mathrm{n} 1$ and $\mathrm{n} 2$ denote the sample size of two distinct stages, respectively. $E_{k}$ and $E_{k}{ }^{\prime}$ denote the Pearson correlation coefficients of the edge $\mathrm{k}$ from the edge E1...Em under two distinct stages, respectively. The real differential score $\mathrm{S}$ was calculated between two stage samples for each module. Subsequently, one thousand random modules were constructed from the same HSSN with the degree conserved. The random differential scores S1...S1000 were also calculated. If the random differential scores were significantly less than the real differential score, the module was considered as a differential stage module (permutation test, $\mathrm{p}$-values $<0.05$ ). The $\mathrm{p}$-values were then adjusted using the Benjamini-Hochberg method. Second, we defined the differential stage modules existing in control/single, control/ recurrent and single/recurrent profiles as disease risk modules to improve the reproducibility. Finally, RRMs were identified from these disease risk modules by the Jonckheere-Terpstra test with gene expression in different stages of samples. The Jonckheere-Terpstra test, which is a non-parametric test, was used to test the distribution of multiple independent samples from the more general ones to find whether there was a significant difference (22). We used the Jonckheere-Terpstra test (http://www.mathworks.com/matlabcentral/fileexchange/ 22159-jonckheere-terpstratest-on-trend/content/jttrend.m) to evaluate the classification significance of RRMs among different stages with a null hypothesis of no differences. The alternative hypothesis was that the sample means changed among the disease stages. A p-value $<0.05$ indicated significant differences among disease stages. The RRMs could reveal the expression differential among control, single and recurrent conditions.

Functional annotation analysis. In order to analyze the biological mechanisms of the RRMs, we applied the geneannotation enrichment analysis to measure the functional characteristics which could increase the possibility to identify the most relevant biological processes (23). Given an RRM with ' $n$ ' genes, the enrichment analysis of the RRM could be efficiently measured by the common and well-known statistical method Hypergeometric test (24). The hypergeometric test is executed for each RRM separately, which is defined by four parameters: i) $\mathrm{N}$ is the size of human genes; ii) $\mathrm{K}$ is the gene number in the annotation terms such as GO terms or pathways; iii) ' $n$ ' is the gene number of the RRM; and iv) ' $c$ ' is the common gene number between the biological annotation terms and the RRM. The hypergeometric test calculates the $\mathrm{p}$-value by using the following formula:

$$
p=\sum_{i=c}^{\min (K, n)} \frac{\left(\begin{array}{l}
K \\
i
\end{array}\right)\left(\begin{array}{l}
N-K \\
n-i
\end{array}\right)}{\left(\begin{array}{l}
N \\
n
\end{array}\right)}
$$

With the p-value $<0.05$ adjusted with Benjamini-Hochberg method, the term is defined as a significant enrichment. The enriched annotation terms in relation to the genes may provide insight into understanding the biology themes behind these genes (25). 
Table I. The recurrent risk modules and disease-related genes for VTE.

\begin{tabular}{|c|c|c|}
\hline RRMs & Genes of the module & Localization \\
\hline M1 & GUCY1A2, GUCY1A3, GUCY1B3, PRKG1, TNNI1, TTN, TCAP, PDE5A, TRIM28, & Cytoplasm \\
\hline M2 & $\begin{array}{l}\text { CEBPD, MXD4, CLU, DDIT3, E2F4, PEG10, GAPDH, MYCBP, LIN28B, LGALS1, } \\
\text { MAX, MYC, NFYB, NFYC, NME2, FBXW7, USP28, KIAA1524, S100A7, SMARCA2, } \\
\text { ZFP36L1, CDC73, TRRAP, CDCA7, MINA, PARP10, PIAS1, DDX18, HN1L, MTDH }\end{array}$ & Nucleus \\
\hline M3 & $\begin{array}{l}\text { TRIM28, PRDX3, CKS1B, DNM1, MTOR, MYCBP, GLS, HDAC1, HMGCS2, HSPD1, HSPE1, } \\
\text { MAOB, MYC, NME1, NME2, GPAM, BCAT1, SHMT1, TFRC, UBE3A, STAM, TRRAP, } \\
\text { CAPZB, TMEM126A, NDUFAF2 }\end{array}$ & Mitochondrion \\
\hline M4 & $\begin{array}{l}\text { CKS2, DLAT, EIF5A, ELAVL1, FDXR, GPX1, GSTP1, HMMR, HNRNPK, TRIAP1, PHB, } \\
\text { DDIT4, BCKDHB, TP53ª , BCL2L14, AIFM2 }\end{array}$ & Mitochondrion \\
\hline M5 & $\begin{array}{l}\text { CPB2, CRYBB2, CTSG, A2M, F2 }{ }^{\mathrm{a}}, \mathrm{F}^{\mathrm{a}}{ }^{\mathrm{a}}, \mathrm{F}^{\mathrm{a}}, \mathrm{F} 8^{\mathrm{a}}, \mathrm{F} 9^{\mathrm{a}}, \mathrm{F} 10, \mathrm{~F} 11, \mathrm{~F} 13 \mathrm{~A} 1^{\mathrm{a}}, \mathrm{F} 13 \mathrm{~B}, \mathrm{FGA}^{\mathrm{a}}, \mathrm{FGB}^{\mathrm{a}} \text {, } \\
\text { FGG, ANGPT1, SERPIND1, SERPINC1 }{ }^{\mathrm{a}}, \mathrm{SERPINA}, \mathrm{PROC}^{\mathrm{a}}, \mathrm{PROS}^{\mathrm{a}}, \mathrm{TFPI}^{\mathrm{a}}, \mathrm{THBD}^{\mathrm{a}}\end{array}$ & Extracell \\
\hline M6 & CXCL13, FGF2, GDF2, SERPINF1, SERPINA4, BGLAP, TNFSF11 & Extracell \\
\hline M7 & $\begin{array}{l}\text { FAS, FASLG, BID, MAP2K4, TNFSF10, FADD, TNFRSF10D, TNFRSF10C, } \\
\text { TNFRSF10B, TNFRSF10A }\end{array}$ & $\begin{array}{l}\text { Plasma } \\
\text { membrane }\end{array}$ \\
\hline
\end{tabular}

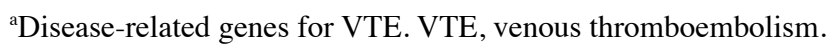

Classifying performance with RRMs. In order to evaluate the classifying performances and recognizing patterns for the RRMs, the Support Vector Machine (SVM) method was employed to discriminate patients at different disease stage $s$ using RRMs as an input feature. Support vector machines are supervised learning models based on relevant learning algorithms (26). The performance of our approach was evaluated using 5-fold cross-validation. The stage sample dataset were equally divided into five parts. We then performed five rounds of cross-validation. One part was used for testing and the others were used for training for each round. We averaged the results of the rounds to obtain the final results. Subsequently, we applied the receiver operating characteristic (ROC) curve to illustrate and evaluate classification performance. The area under the ROC curve (AUC) quantifies the overall discriminative power of the test (27).

\section{Results}

RRMs of VTE. The differential stage modules were obtained by assessing the significantly expression changes in the gene expression profile datasets. The disease risk modules were screened out. Finally, seven RRMs were identified through the Jonckheere-Terpstra test (Table I).

The functional characteristics of the RRMs. The correlation between RRMs and VTE was investigated by a literature review and functional annotation analysis (28). The functional categories were mostly enriched in the RRMs were coagulation cascades, blood circulation, signal transduction and cell death (Table II), and the coagulation cascades pathway, apoptosis and p53 signaling pathway (Fig. 1A).

Previous studies have demonstrated that these annotated functional categories and pathways are associated with VTE. For example, it has been shown that the alterations in both
Table II. The functional category of recurrent risk modules.

\begin{tabular}{lcccc}
\hline & \multicolumn{4}{c}{ Functional category } \\
\cline { 2 - 5 } Module & Cell death & $\begin{array}{c}\text { Coagulation } \\
\text { cascades }\end{array}$ & $\begin{array}{c}\text { Blood } \\
\text { circulation }\end{array}$ & $\begin{array}{c}\text { Signal } \\
\text { transduction }\end{array}$ \\
\hline M1 & - & - & $\sqrt{ }$ & - \\
M2 & - & - & - & $\sqrt{ }$ \\
M3 & $\sqrt{ }$ & - & - & - \\
M4 & $\sqrt{ }$ & - & - & - \\
M5 & - & $\sqrt{ }$ & $\sqrt{ }$ & $\sqrt{ }$ \\
M6 & $\sqrt{ }$ & - & - & - \\
M7 & $\sqrt{ }$ & - & - & $\sqrt{ }$ \\
\hline
\end{tabular}

Modules which are marked with a tick $(\sqrt{ })$ indicate that the respective module possesses the corresponding functional category. Modules which are marked with a dahs (-) indicate that the respective module does not possess the corresponding functional category.

the complement and coagulation systems are associated with VTE $(29,30)$. The coagulation cascade is a key component of the hemostatic process and limits blood loss in response to tissue injury. Derangements in this process can lead to VTE (31). Gawaz and Vogel indicated that apoptosis plays a critical role in platelet life and is implicated in the pathogenicity of thrombosis (32).

Classifying the capacity of RRMs. With the rapid development of systems biology, the modular analysis of biological networks may allow for the better understanding of the molecular mechanisms responsible for disease and may aid researchers in the identification of multiple module 
Table III. Five-fold cross-validation for the RRMs of VTE in the HSSNs.

\begin{tabular}{lllll}
\hline $\begin{array}{l}\text { Classification } \\
\text { features }\end{array}$ & \multicolumn{1}{c}{ Datasets } & AUC & TPR & FPR \\
\hline RRMs & Recurrent/control & 0.8584 & 0.9206 & 0.2632 \\
& Recurrent/single & 0.8118 & 0.8269 & 0.25 \\
& Single/control & 0.7561 & 0.9841 & 0.4737 \\
M4 & Recurrent/control & 0.8084 & 0.75 & 0.1842 \\
M5 & Recurrent/control & 0.868 & 0.8254 & 0.1842 \\
M7 & Recurrent/control & 0.8129 & 0.8571 & 0.1579
\end{tabular}

VTE, venous thromboembolism; RRMs, recurrent risk modules; HSSNs, human subcellular signaling networks; FPR, false positive rate; TPR, true positive rate; AUC, area under the curve.

biomarkers (33). SVM can be used to identify recurrentrelated patients and evaluate the classifying performance with RRMs as a classification feature. The average expression values of RRMs were the classification features of SVM classifier to distinguish patients with different stages of VTE. We performed a 5-fold cross-validation on VTE stage samples to evaluate the discriminative power of RRMs. The AUC values of the 5-fold cross-validation for each twostage samples (recurrent/control, recurrent/single and single/ control) were evaluated, respectively (Fig. 1B). As a result, the high discriminative power was obtained using RRMs as classification features (Table III).

In order to further evaluate the effectiveness of our RRMs, the recurrent/control samples were used for training, and the recurrent/single and single/control samples were used for testing in the GSE19151 dataset. The AUC values derived from this model were 0.9062 and 0.7577 , respectively (Table IV). The high discriminative power suggested that the patients could be effectively distinguished using RRMs as classification features.

Furthermore, each RRM from the individual datasets as classification features was used to construct a classifier to recognize patients with recurrent disease. The discriminative power of each RRM was evaluated between different stages of VTE patients with the 5-fold across-validation. Moreover, the recurrent/control samples were used for training and the single/control and recurrent/single samples were used for testing in the GSE19151 dataset for each RRM as classification features. We found that the RRMs M4, M5 and M7 had good classification performance to discriminate patients wtih recurrent disease (Tables III and IV). In particular, RRM M5 had the best discriminative power among the RRMs. This indicated that M5 may be used to construct an individual classifier to efficiently distinguish the patients with recurrent disease.

The robustness and stability of our algorithm. Naive Bayes and random forests were used to evaluate the robustness and stability of our algorithm, which also considered the RRM expression values as the classification features to confirm the high classification performance for VTE stages. The Naive Bayes classifier is based on Bayes' theorem with independence assumptions between predictors (34). Random forests are an ensemble learning method for classification and regression that fits a multitude of decision tree classifiers on various sub-samples of the dataset and uses averaging to improve the predictive accuracy (35). The RRMs had a high classification performance and stability, by not only Naive Bayes but also random forests (Table V).

To better evaluate the discriminative power of the RRMs for other independent datasets, the recurrent/control samples of GSE19151 were used for training, and the VT sample/normal samples of GSE17078 were used for testing (Table VI). High AUC values were presented in the VTE stage datasets (the AUC values were 0.88 to 0.83 ). The results indicated that the RRMs had a good classification performance for the independent datasets. In a word, the RRMs identified by our algorithm with high AUC values have a strong discriminative power, robustness and stability.

Comparison with average expression value method. The expression correlation method was compared with the average expression value method. The RRMs for VTE were identified using the average expression value method. Supposing a module $\mathrm{M}^{\prime}$ contains $\mathrm{G}$ genes thus the average expression value differential score $\mathrm{S}^{\prime}$ was expressed as follows:

$$
S^{\prime}\left(\mathrm{M}^{\prime}\right)=\sum_{k=1}^{G}\left|\frac{\sum_{i=1}^{U} Z_{i}}{U}-\frac{\sum_{j=1}^{V} Z^{\prime}}{V}\right|
$$

where $\mathrm{Z}$ and $\mathrm{Z}^{\prime}$ denote the expression values of genes in two distinct stages, respectively, $\mathrm{U}$ and $\mathrm{V}$ denote the numbers of samples in two distinct stages, respectively. For a network module, we calculated the real average expressed differential score S'. Subsequently, one thousand random modules were constructed from the same HSSN with the degree conserved. The random average expression value differential scores $\mathrm{S}^{\prime} 1$...S'1000 were also calculated. If the random average expression value differential scores were significantly less than the real average expressed differential score, the module was considered as an average expression value differential stage module (permutation test, $\mathrm{p}$-values $<0.05$ ). The p-values were adjusted using the Benjamini-Hochberg method. We then defined the average expression value differential stage modules existing in control/single, control/recurrent and single/recurrent profiles as the average expression value disease risk modules to improve the reproducibility.

However, we could not find the average expression value disease risk modules among the VTE stage samples. The result suggested that expression correlation method outperformed the average expression value method, and may better reflect the difference between VTE stage samples.

The drug target characteristics of the RRMs. The DrugBank database is a comprehensive, high-quality, freely accessible and online bioinformatics and cheminformatics resource, which contains detailed information about drugs and drug targets (36). The drug targets associated with the pathogenesis of VTE were searched from the DrugBank database and mapped into the RRMs. Nine approved drug targets were 
Table IV. Classification performances for the RRMs of VTE in HSSNs.

\begin{tabular}{|c|c|c|c|c|c|}
\hline $\begin{array}{l}\text { Classification } \\
\text { features }\end{array}$ & Train set & Test set & AUC & TPR & FPR \\
\hline \multirow[t]{2}{*}{ RRMs } & Recurrent/control & Recurrent/single & 0.9062 & 0.9048 & 0.1875 \\
\hline & Recurrent/control & Single/control & 0.7577 & 0.9683 & 0.4737 \\
\hline \multirow[t]{2}{*}{ M5 } & Recurrent/control & Recurrent/single & 0.872 & 0.9524 & 0.2188 \\
\hline & Recurrent/control & Single/control & 0.7657 & 0.9524 & 0.4737 \\
\hline
\end{tabular}

RRMs, recurrent risk modules; VTE, venous thromboembolism; HSSNs, human subcellular signaling networks; FPR, false positive rate; TPR, true positive rate; AUC, area under the curve.

Table V. Five-fold cross-validation for the RRMs of VTE using Naive Bayes and Random Forest.

\begin{tabular}{|c|c|c|c|c|c|}
\hline Classifier & Features & Datasets & AUC & TPR & FPR \\
\hline Naive Bayes & RRMs & Recurrent/control & 0.8496 & 0.8571 & 0.2368 \\
\hline Naive Bayes & M5 & Recurrent/control & 0.8634 & 0.9048 & 0.2105 \\
\hline Random Forest & RRMs & Recurrent/control & 0.8375 & 0.7937 & 0.1842 \\
\hline Random Forest & M5 & Recurrent/control & 0.8488 & 0.8889 & 0.2368 \\
\hline
\end{tabular}

RRMs, recurrent risk modules; VTE, venous thromboembolism; FPR, false positive rate; TPR, true positive rate; AUC, area under the curve.

Table VI. Classification performances for the RRMs for the independent datasets.

\begin{tabular}{lllrr}
\hline $\begin{array}{l}\text { Classification } \\
\text { features }\end{array}$ & Train set & Test set & AUC & TPR \\
\hline RRMs & Recurrent/control & VT sample/normal & 0.83 & 0.9683 \\
M5 & Recurrent/control & VT sample/normal & 0.8828 & 0.3421 \\
\hline
\end{tabular}

RRMs, recurrent risk modules; FPR, false positive rate; TPR, true positive rate; AUC, area under the curve.

Table VII. The approved drug targets and therapeutic drugs of VTE in the recurrent risk modules.

\begin{tabular}{|c|c|c|}
\hline Modules & Drug targets & Drug \\
\hline M1 & PDE5A & Dipyridamole \\
\hline M5 & $\mathrm{F} 2$ & Argatroban, bivalirudin, dabigatran etexilate, lepirudin, warfarin \\
\hline M5 & F10 & $\begin{array}{l}\text { Apixaban, certoparin sodium, edoxaban, enoxaparin sodium, fondaparinux sodium, } \\
\text { heparin, otamixaban, rivaroxaban }\end{array}$ \\
\hline M5 & FGA & Alteplase, anistreplase, reteplase \\
\hline M5 & SERPIND1 & Ardeparin sodium, sulodexide \\
\hline M5 & SERPINC1 & $\begin{array}{l}\text { Ardeparin sodium, certoparin sodium, dalteparin, dalteparin sodium, enoxaparin sodium, } \\
\text { fondaparinux sodium, heparin, nadroparin, nadroparin calcium, sulodexide, tinzaparin sodium }\end{array}$ \\
\hline M5 & SERPINA5 & Urokinase \\
\hline M5 & TFPI & Dalteparin sodium \\
\hline M6 & FGF2 & Phenprocoumon \\
\hline
\end{tabular}

VTE, venous thromboembolism. 


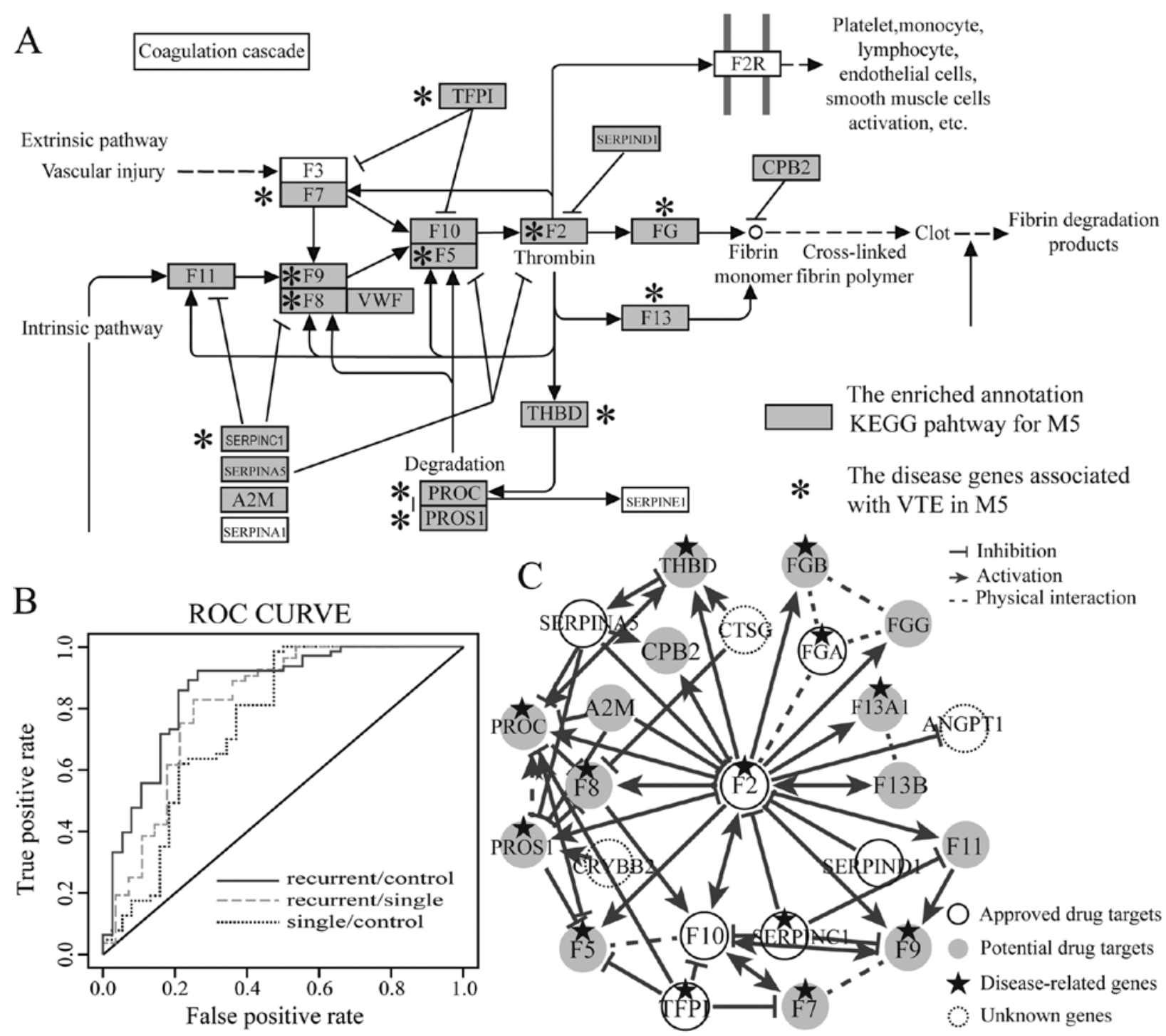

Figure 1. (A) The enriched KEGG pathways for the M5. The venous thromboembolism (VTE) disease genes are marked by an asterisk (*). (B) Five-fold crossvalidation for GSE19151 recurrent/control, recurrent/single and single/control for the recurrent risk modules (RRMs) of VTE in the human subcellular signaling networks (HSSNs). (C) The genes and drug targets in M5 for recurrent VTE.

identified in the RRMs (Table VII). In particular, seven drug targets for VTE treatment are in M5 and are targeted by many of the drugs. M5 has the significant enrichment characteristics for the drug targets ( $p$-value $<0.01$ ).

The drug target [coagulation factor II, thrombin (F2)] of warfarin plays crucial role in M5, interacting with 19 VTE disease-related genes (Fig. 1C). In addition, F2 is the drug target of other therapeutic drugs for VTE such as argatroban, dabigatran and bivalirudin. Seven of the genes that interacted with F2 were the approved therapeutic drug targets for VTE, revealing that these genes played an important role in the treatment of recurrent VTE. Moreover, our results revealed that M5 not only had a high discriminative power, but also had significant function associated with the pathogenesis of VTE. Therefore, we defined the genes coagulation factor XI (F11), alpha-2-macroglobulin (A2M), coagulation factor XIII A chain (F13A1), coagulation factor VIII (F8), coagulation factor IX (F9), coagulation factor VII (F7), protein C, inactivator of coagulation factors Va and VIIIa (PROC), coagulation factor XIII B chain (F13B), fibrinogen gamma chain (FGG), thrombo- modulin (THBD), coagulation factor V (F5), fibrinogen beta chain (FGB), protein $\mathrm{S}$ (alpha) (PROS1), carboxypeptidase B2 (CPB2) in M5 and the RRM M5 itself as potential drug targets (Fig. 1C). These potential drug targets would be promising to further research, exploiting new therapeutic drugs to treat VTE patients.

\section{Discussion}

In this study, we developed a novel strategy which combined both dynamic gene expression and the human subcellular signaling networks to identify RRMs. Seven RRMs in the HSSNs were identified and mainly annotated in blood coagulation and apoptosis, which were strongly associated with the pathogenesis of VTE $(29,30)$. Norris pointed out that the activation of coagulation may predispose affected individuals to thrombosis (37). White and Kile indicated that the platelet activation responses contained components of apoptotic machinery which are of particular importance for thrombus formation, which highlighted a potential role in apoptotic 
Table VIII. The enriched KEGG pathway for RRMs.

\begin{tabular}{|c|c|c|c|}
\hline RRM & KEGG pathway & The enriched genes & Benjamini \\
\hline M1 & Long-term depression & GUCY1A2, GUCY1A3, GUCY1B3, PRKG1 & $1.64 \mathrm{E}-04$ \\
\hline M1 & Gap junction & GUCY1A2, GUCY1A3, GUCY1B3, PRKG1 & $1.77 \mathrm{E}-04$ \\
\hline M1 & $\begin{array}{l}\text { Vascular smooth muscle } \\
\text { contraction }\end{array}$ & GUCY1A2, GUCY1A3, GUCY1B3, PRKG1 & $2.35 \mathrm{E}-04$ \\
\hline M1 & Purine metabolism & PDE5A, GUCY1A2, GUCY1A3, GUCY1B3 & 4.47E-04 \\
\hline M5 & $\begin{array}{l}\text { Complement and } \\
\text { coagulation cascades }\end{array}$ & $\begin{array}{l}\text { F11, A2M, F10, F13A1, F8, F9, F7, PROC, F13B, FGG, THBD, } \\
\text { F5, FGA, FGB, SERPINA5, F2, SERPINC1, TFPI, } \\
\text { SERPIND1, PROS1, CPB2 }\end{array}$ & $2.73 \mathrm{E}-37$ \\
\hline M7 & Apoptosis & $\begin{array}{l}\text { TNFRSF10A, BID, TNFSF10, TNFRSF10C, TNFRSF10B, TNFRSF10D, } \\
\text { FASLG, FADD, FAS }\end{array}$ & $9.41 \mathrm{E}-13$ \\
\hline M7 & $\begin{array}{l}\text { Natural killer cell } \\
\text { mediated cytotoxicity }\end{array}$ & $\begin{array}{l}\text { TNFRSF10A, BID, TNFSF10, TNFRSF10C, TNFRSF10B, } \\
\text { TNFRSF10D, FASLG, FAS }\end{array}$ & $2.47 \mathrm{E}-09$ \\
\hline M7 & $\begin{array}{l}\text { Cytokine-cytokine } \\
\text { receptor interaction }\end{array}$ & $\begin{array}{l}\text { TNFRSF10A, TNFSF10, TNFRSF10C, TNFRSF10B } \\
\text { TNFRSF10D, FASLG, FAS }\end{array}$ & 8.69E-06 \\
\hline M7 & p53 signaling pathway & BID, TNFRSF10B, FAS & 0.02949 \\
\hline
\end{tabular}

RRMs, recurrent risk modules.

processes of thrombosis (38). Moreover, our algorithm had a good classification performance, robustness and stability. The RRMs had strong discriminative power to distinguish patients in different stages of disease development. In particulaar, the M5 had the best performance. It was suggested that the M5 was effective in elucidating the pathogenesis of VTE, which holds promise for the development of therapeutic strategies for the treatment of VTE.

Warfarin is an effective antithrombotic agent, but noncompliance and cessation with warfarin therapy for over a year are associated with a higher risk of recurrent VTE and the therapeutic range is narrow (39). Our results revealed that nine approved drug targets in the RRMs were targeted not only by warfarin, but also by the other therapeutic drugs (such as apixaban and dabigatran) for VTE. In particular, seven approved drug target genes in the M5 were targeted by three types of drugs (anticoagulant drugs, thrombolytic drugs and antiplatelet drugs) for the treatment of VTE. For example, F2 is the antithrombotic and anticoagulant drug target of not only warfarin, but also dabigatran, argatroban, lepirudin and bivalirudin. Coagulation factor X (F10) is the common anticoagulant drug target of apixaban, edoxaban, heparin and enoxaparin sodium.

Among the identified RRM modules, M5 had the best discriminative power. Thirteen disease-related genes in M5 are associated with the pathogenesis of VTE from OMIM (http:// www.omim.org/) and Genetic Association Database (http:// geneticassociationdb.nih.gov/) (Table I). The genes, serpin family C member 1 (SERPINC1), F2, F7 and fibrinogen alpha chain (FGA), were not only the disease-related genes, but also the approved drug targets. The gene SERPINC1 encodes antithrombin III, which is a major risk factor of VTE, acting as an inhibitor of thrombin and other coagulation proteinases (40). Many new drugs, such as dabigatran and argatroban, targeting F2 may be used as effective anticoagulats for the treatment of recurrent VTE (41). The genes, F10, serpin family A member 5 (SERPINA5) and serpin family D member 1 (SERPIND1), were the approved drug targets. The new oral anticoagulant drugs, rivaroxaban, apixaban and edoxaban, which are direct inhibitors of F10, have been the approved drugs or have entered clinical practice for different clinical indications (1). The gene, F5, F7, F8, F9 and PROC, are both disease-related genes and the potential drug targets. The genes, F5, F9 and PROC, are involved in the blood coagulation pathway. The dysfunction of these is associated with VTE (42). The combination of F5 with F10 activates F2 to form the effector enzyme of the coagulation cascade (43), and the dysfunction of this contributes to VTE $(4,44)$.

Multiple therapeutic modes and options exist for VTE treatment. The prevention and initial therapy of VTE usually begin with the administration of rapidly acting parenteral anticoagulants, such as heparin (45). Furthermore, vitamin K antagonists (e.g., warfarin) are the commonly available oral anticoagulant therapy used for decades (46). However, the risk of recurrent VTE also exists following treatment with heparin and warfarin $(47,48)$. In the study by Deitelzweig et al, it was demonstrated that the cessation of warfarin therapy in 1,027 patients within three months led to recurrence in $915(89.1 \%)$ patients (48). In order to treat the recurrence, alternative drugs and drug combination therapy should be considered, as well as the drugs for extended VTE treatment $(10,49)$. Long-term anticoagulation with low molecular weight heparin (LMWH) compared with warfarin appears to be more effective for the prevention of recurrent VTE (50). Compared with warfarin, dabigatran does not require close monitoring and the adjusting of dosage for extended therapy and has a lower rate of major or clinically relevant bleeding (51). Pillai et al described that 
therapy with dalteparin combined with warfarin effectively prevented recurrent VTE (49). Furthermore, these effective therapeutic drugs consistently target the crucial drug targets in M5, which is significantly associated with VTE.

In total, $87.5 \%$ of the genes in the M5 were significantly enriched in blood coagulation pathway. The analysis of drug targets in M5 may contribute to elucidating the pathogenesis of VTE. Furthermore, multiple drug targets and multi therapeutic drugs should be considered for VTE therapy. New therapeutic drugs may be explored by the analysis of VTE potential drug targets, such as F5, F7, F8, F9, which may hold promise for improving the safety and effectiveness of VTE treatment (2). In addition, the M1 and M7 were significantly enriched in the VTE-related pathway, which may bring new insight into VTE research, as well as the therapeutic drug targets identification (Table VIII). For instance, a recent study indicated that atherosclerosis, thrombosis and myocardial infarction were significantly associated with vascular smooth muscle contraction and the apoptotic pathway (52). Freedman suggested that GUCY1A3 mutations were related to thrombosis (53). Zeng et al indicated that miR-20a regulates the PRKG1 gene, thereby promoting the proliferation, and contributes to thrombosis (54). TNF receptor superfamily members 10B, 10C, 10D and TNFSF10 linked to inflammation and coagulation and contribute to VTE (55). Although the potential drug targets identified by our computational biology algorithm are just validated by the literature review theoretically, the treatment effectiveness of these should be validated by experimental studies.

It is noteworthy that the differentially expressed genes were commonly considered as the classification features to discriminate patients or disease states. However, the differentially expressed genes were not identified between different stages of the VTE samples, which could not be used to identify patients with recurrent disease. The RRMs identified with the expression dynamic changes of the gene had a better advantage for recurrent VTE treatment.

In conclusion, signaling proteins or gene products may play a unique role in biological activity and may be intriguing to investigators in both basic studies and drug development. The RRMs based on the signaling network were identified with dynamic changes related to VTE recurrence from the system level. The RRMs (particularly M5) not only implied a good discriminative power to distinguish recurrent patients, but also had the crucial drug targets for VTE treatment. Most genes in M5 were proofed associated with VTE by the literature review. This suggested that the M5 may provide an effective guidance for the investigation of the mechanisms responsible for recurrent VTE, and for therapeutic drug screening and pharmaceutical studies.

\section{Acknowledgements}

This study was supported in part by the Science and Technology Research Project of the National Natural Science Foundation of China (grant nos. 61272388 and 31301040); the Natural Science Foundation of Heilongjiang Province (grant no. F201237); the Science and Technology Research Project of the Heilongjiang Ministry of Education (grant no. 12541476); the Science Foundation of Heilongjiang Health Department (grant no. 2012-810); and the Master Innovation Funds of Heilongjiang Province (grant no. YJSCX2014-18HYD).

\section{References}

1. Kyrle PA and Eichinger S: Clinical scores to predict recurrence risk of venous thromboembolism. Thromb Haemost 108: 1061-1064, 2012

2. Becattini $\mathrm{C}$ and Manina G: Long-term treatment of venous thromboembolism. Curr Vasc Pharmacol 12: 384-392, 2014.

3. Hogg K, Wells PS and Gandara E: The diagnosis of venous thromboembolism. Semin Thromb Hemost 38: 691-701, 2012.

4. Pabinger I, Thaler J and Ay C: Biomarkers for prediction of venous thromboembolism in cancer. Blood 122: 2011-2018, 2013.

5. Perry JR: Thromboembolic disease in patients with high-grade glioma. Neuro Oncol 14 (Suppl 4): iv73-iv80, 2012.

6. Mackman N: New insights into the mechanisms of venous thrombosis. J Clin Invest 122: 2331-2336, 2012.

7. Wells PS, Forgie MA and Rodger MA: Treatment of venous thromboembolism. JAMA 311: 717-728, 2014.

8. Kearon C: Long-term anticoagulation for venous thromboembolism: Duration of treatment and management of warfarin therapy. Clin Chest Med 31: 719-730, 2010.

9. Zhu T, Martinez I and Emmerich J: Venous thromboembolism: Risk factors for recurrence. Arterioscler Thromb Vasc Biol 29: 298-310, 2009.

10. Sharifi M, Bay C, Schwartz F and Skrocki L: Safe-dose thrombolysis plus rivaroxaban for moderate and severe pulmonary embolism: Drip, drug, and discharge. Clin Cardiol 37: 78-82, 2014.

11. Barabási AL and Oltvai ZN: Network biology: Understanding the cell's functional organization. Nat Rev Genet 5: 101-113, 2004.

12. Suthram S, Dudley JT, Chiang AP, Chen R, Hastie TJ and Butte AJ: Network-based elucidation of human disease similarities reveals common functional modules enriched for pluripotent drug targets. PLoS Comput Biol 6: e1000662, 2010.

13. Laenen G, Thorrez L, Börnigen D and Moreau Y: Finding the targets of a drug by integration of gene expression data with a protein interaction network. Mol Biosyst 9: 1676-1685, 2013.

14. Martinelli I, De Stefano V and Mannucci PM: Inherited risk factors for venous thromboembolism. Nat Rev Cardiol 11: 140-156, 2014

15. Newman RH, Hu J, Rho HS, Xie Z, Woodard C, Neiswinger J, Cooper C, Shirley M, Clark HM, Hu S, et al: Construction of human activity-based phosphorylation networks. Mol Syst Biol 9: 655, 2013.

16. Li L, Tibiche C, Fu C, Kaneko T, Moran MF, Schiller MR, Li SS and Wang E: The human phosphotyrosine signaling network: Evolution and hotspots of hijacking in cancer. Genome Res 22: 1222-1230, 2012

17. Ashburner M, Ball CA, Blake JA, Botstein D, Butler H, Cherry JM, Davis AP, Dolinski K, Dwight SS, Eppig JT, et al; The Gene Ontology Consortium: Gene ontology: Tool for the unification of biology. Nat Genet 25: 25-29, 2000.

18. Magrane M and Consortium U: UniProt Knowledgebase: A hub of integrated protein data. Database (Oxford) 2011: bar009, 2011.

19. Samsa G, Matchar DB, Dolor RJ, Wiklund I, Hedner E, Wygant G, Hauch O, Marple CB and Edwards R: A new instrument for measuring anticoagulation-related quality of life: development and preliminary validation. Health Qual Life Outcomes 2: 22 , 2004.

20. Lewis DA, Stashenko GJ, Akay OM, Price LI, Owzar K, Ginsburg GS, Chi JT and Ortel TL: Whole blood gene expression analyses in patients with single versus recurrent venous thromboembolism. Thromb Res 128: 536-540, 2011.

21. Reimand J, Tooming L, Peterson H, Adler P and Vilo J: GraphWeb: Mining heterogeneous biological networks for gene modules with functional significance. Nucleic Acids Res 36: W452-W459, 2008

22. Bewick V, Cheek L and Ball J: Statistics review 10: Further nonparametric methods. Crit Care 8: 196-199, 2004.

23. Huang W, Sherman BT and Lempicki RA: Systematic and integrative analysis of large gene lists using DAVID bioinformatics resources. Nat Protoc 4: 44-57, 2009.

24. Huang W, Sherman BT and Lempicki RA: Bioinformatics enrichment tools: Paths toward the comprehensive functional analysis of large gene lists. Nucleic Acids Res 37: 1-13, 2009.

25. Curtis RK, Oresic M and Vidal-Puig A: Pathways to the analysis of microarray data. Trends Biotechnol 23: 429-435, 2005.

26. Mukherjee K, Abhipriya, Vidyarthi AS and Pandey DM: SVM based model generation for binding site prediction on helix turn helix motif type of transcription factors in eukaryotes. Bioinformation 9: 500-505, 2013. 
27. Bradley AP: The use of the area under the ROC curve in the evaluation of machine learning algorithms. Pattern Recognition 30: 1145-1159, 1997.

28. Dennis G Jr, Sherman BT, Hosack DA, Yang J, Gao W, Lane HC and Lempicki RA: DAVID: Database for annotation, visualization, and integrated discovery. Genome Biol 4: P3, 2003.

29. Nojima J, Suehisa E, Kuratsune H, Machii T, Koike T, Kitani T, Kanakura Y and Amino N: Platelet activation induced by combined effects of anticardiolipin and lupus anticoagulant $\operatorname{IgG}$ antibodies in patients with systemic lupus erythematosus - possible association with thrombotic and thrombocytopenic complications. Thromb Haemost 81: 436-441, 1999.

30. Inanc M, Donohoe S, Ravirajan CT, Radway-Bright EL, Mackie I, Machin S and Isenberg DA: Anti-beta2-glycoprotein I anti-prothrombin and anticardiolipin antibodies in a longitudinal study of patients with systemic lupus erythematosus and the antiphospholipid syndrome. Br J Rheumatol 37: 1089-1094, 1998

31. Markiewski MM, Nilsson B, Ekdahl KN, Mollnes TE and Lambris JD: Complement and coagulation: Strangers or partners in crime? Trends Immunol 28: 184-192, 2007.

32. Gawaz M and Vogel S: Platelets in tissue repair: Control of apoptosis and interactions with regenerative cells. Blood 122 2550-2554, 2013.

33. Meunier D, Fonlupt P, Saive AL, Plailly J, Ravel N and Royet JP: Modular structure of functional networks in olfactory memory. Neuroimage 95: 264-275, 2014.

34. Maruyama O: Heterodimeric protein complex identification by naïve Bayes classifiers. BMC Bioinformatics 14: 347, 2013.

35. Azar AT, Elshazly HI, Hassanien AE and Elkorany AM: A random forest classifier for lymph diseases. Comput Methods Programs Biomed 113: 465-473, 2014.

36. Wishart DS, Knox C, Guo AC, Cheng D, Shrivastava S, Tzur D, Gautam B and Hassanali M: DrugBank: A knowledgebase for drugs, drug actions and drug targets. Nucleic Acids Res 36: D901-D906, 2008.

37. Norris LA: Blood coagulation. Best Pract Res Clin Obstet Gynaecol 17: 369-383, 2003.

38. White MJ and Kile BT: Apoptotic processes in megakaryocytes and platelets. Semin Hematol 47: 227-234, 2010.

39. Thomé S: ACP Journal Club. Edoxaban was noninferior to warfarin for preventing recurrent venous thromboembolism, with less bleeding. Ann Intern Med 160: JC4, 2014.

40. Lane DA, Olds RJ and Thein SL: Antithrombin III: Summary of first database update. Nucleic Acids Res 22: 3556-3559, 1994.

41. Hull RD and Gersh MH: The current landscape of treatment options for venous thromboembolism: A focus on novel oral anticoagulants. Curr Med Res Opin 31: 197-210, 2014.

42. Eikelboom JW, Zelenkofske SL and Rusconi CP: Coagulation factor IXa as a target for treatment and prophylaxis of venous thromboembolism. Arterioscler Thromb Vasc Biol 30: 382-387, 2010 .
43. Cripe LD, Moore KD and Kane WH: Structure of the gene for human coagulation factor V. Biochemistry 31: 3777-3785, 1992.

44. Hoyer LW and Hemophilia A: Hemophilia A. N Engl J Med 330: 38-47, 1994.

45. Geerts WH, Bergqvist D, Pineo GF, Heit JA, Samama CM, Lassen MR and Colwell CW; American College of Chest Physicians: Prevention of venous thromboembolism: American College of Chest Physicians Evidence-Based Clinical Practice Guidelines (8th Edition). Chest 133 (Suppl 6): 381S-453S, 2008.

46. Abo-Salem E and Becker R: Transitioning to and from the novel oral anticoagulants: A management strategy for clinicians. J Thromb Thrombolysis 37: 372-379, 2014.

47. Chee CE, Ashrani AA, Marks RS, Petterson TM, Bailey KR, Melton LJ III and Heit JA: Predictors of venous thromboembolism recurrence and bleeding among active cancer patients: A population-based cohort study. Blood 123: 3972-3978, 2014.

48. Deitelzweig SB, Lin J, Kreilick C, Hussein M and Battleman D: Warfarin therapy in patients with venous thromboembolism: Patterns of use and predictors of clinical outcomes. Adv Ther 27: 623-633, 2010

49. Pillai AR, Olujohungbe A, Evans MR, Main NJ and Hunt BJ: The management of recurrent VTE in cancer patients receiving therapeutic anticoagulation: The use of dual anticoagulant therapy combined with an IVC filter. Blood Coagul Fibrinolysis 21: 766-769, 2010

50. Akl EA, Kahale L, Barba M, Neumann I, Labedi N, Terrenato I, Sperati F, Muti P and Schünemann H: Anticoagulation for the long-term treatment of venous thromboembolism in patients with cancer. Cochrane Database Syst Rev 7: CD006650, 2014.

51. Schulman S, Kearon C, Kakkar AK, Schellong S, Eriksson H, Baanstra D, Kvamme AM, Friedman J, Mismetti P and Goldhaber SZ; RE-MEDY Trial Investigators; RE-SONATE Trial Investigators: Extended use of dabigatran, warfarin, or placebo in venous thromboembolism. N Engl J Med 368: 709-718, 2013.

52. Yu Y, Qin J, Chen D, Wang H, Wang J and Yu Y: Chronic cardiovascular disease-associated gene network analysis in human umbilical vein endothelial cells exposed to $2,3,7,8$-tetrachlorodibenzo-p-dioxin. Cardiovasc Toxicol 15: 157-171, 2015.

53. Freedman JE: Inherited dysfunctional nitric oxide signaling and the pathobiology of atherothrombotic disease. Circ Res 114 1372-1373, 2014.

54. Zeng Y, Pan Y, Liu H, Kang K, Wu Y, Hui G, Peng W, Ramchandran R, Raj JU and Gou D: MiR-20a regulates the PRKG1 gene by targeting its coding region in pulmonary arterial smooth muscle cells. FEBS Lett 588: 4677-4685, 2014.

55. Lv W, Duan Q, Wang L, Gong Z, Yang F and Song Y: Gene expression levels of cytokines in peripheral blood mononuclear cells from patients with pulmonary embolism. Mol Med Rep 7: 1245-1250, 2013 